\title{
The genetic basis of tail-loss evolution in humans and apes
}

4 Bo Xia ${ }^{1,2 *}$, Weimin Zhang ${ }^{2}$, Aleksandra Wudzinska², Emily Huang ${ }^{2}$, Ran Brosh², Maayan Pour ${ }^{1}$, Alexander Miller ${ }^{4}$, Jeremy S. Dasen ${ }^{4}$, Matthew T. Maurano ${ }^{2}$, Sang Y. Kim ${ }^{5}$, Jef D. Boeke ${ }^{2,3,6 *}$

6 and Itai Yanai ${ }^{1,3 *}$

8

${ }^{1}$ Institute for Computational Medicine, NYU Langone Health, New York, NY 10016, USA

10

${ }^{2}$ Institute for Systems Genetics, NYU Langone Health, New York, NY 10016, USA

${ }^{3}$ Department of Biochemistry and Molecular Pharmacology, NYU Langone Health, New York,

12 NY 10016, USA

${ }^{4}$ Department of Neuroscience and Physiology, NYU Langone Health, New York, NY 10016,

14 USA

${ }^{5}$ Department of Pathology, NYU Langone Health, New York, NY 10016, USA

$16{ }^{6}$ Department of Biomedical Engineering, NYU Tandon School of Engineering, Brooklyn, NY, 11201, USA

18

*Correspondence: Bo.Xia@nyulangone.edu; Jef.Boeke@nyulangone.org;

20 Itai.Yanai@nyulangone.org 
The loss of the tail is one of the main anatomical evolutionary changes to have occurred

2 along the lineage leading to humans and to the "anthropomorphous apes"1,2. This morphological reprogramming in the ancestral hominoids has been long considered to

4 have accommodated a characteristic style of locomotion and contributed to the evolution of bipedalism in humans ${ }^{3-5}$. Yet, the precise genetic mechanism that facilitated tail-loss

6 evolution in hominoids remains unknown. Primate genome sequencing projects have made possible the identification of causal links between genotypic and phenotypic

8 changes $^{6-8}$, and enable the search for hominoid-specific genetic elements controlling tail development ${ }^{9}$. Here, we present evidence that tail-loss evolution was mediated by the insertion of an individual Alu element into the genome of the hominoid ancestor. We demonstrate that this Alu element - inserted into an intron of the TBXT gene (also called T or Brachyury ${ }^{10-12}$ ) - pairs with a neighboring ancestral Alu element encoded in the reverse genomic orientation and leads to a hominoid-specific alternative splicing event.

14 To study the effect of this splicing event, we generated a mouse model that mimics the expression of human TBXT products by expressing both full-length and exon-skipped isoforms of the mouse TBXT ortholog. We found that mice with this genotype exhibit the complete absence of a tail or a shortened tail, supporting the notion that the exonskipped transcript is sufficient to induce a tail-loss phenotype, albeit with incomplete penetrance. We further noted that mice homozygous for the exon-skipped isoforms exhibited embryonic spinal cord malformations, resembling a neural tube defect condition, which affects $\sim 1 / 1000$ human neonates ${ }^{13}$. We propose that selection for the

22 loss of the tail along the hominoid lineage was associated with an adaptive cost of potential neural tube defects and that this ancient evolutionary trade-off may thus 24 continue to affect human health today. 
The tail appendage varies widely in its morphology and function across vertebrate species ${ }^{4,5}$.

2 For primates in particular, the tail is adapted to a range of environments, with implications for the animal's style of locomotion ${ }^{14,15}$. The New World howler monkeys, for example, evolved a

4 prehensile tail that helps the animal to grasp or hold objects while occupying arboreal habitats ${ }^{16}$. Hominoids - which include humans and the apes - however, are distinct among the primates in

6 their loss of an external tail (Fig. 1a). The loss of the tail is inferred to have occurred $\sim 25$ million years ago when the hominoid lineage diverged from the ancient Old World monkeys (Fig. 1a),

8 leaving only 3-4 caudal vertebrae to form the coccyx, or tailbone, in modern humans ${ }^{17}$. It has long been speculated that tail loss in hominoids has contributed to bipedal locomotion, whose evolutionary occurrence coincided with the loss of tail ${ }^{18-20}$. Recent progress in developmental biology has led to the elucidation of the gene regulatory networks that underlie tail development $^{9,21}$. Specifically, the absence of the tail phenotype in the Mouse Genome Informatics has so far recorded 31 genes from the study of mutants and naturally occurring variants $^{21,22}$ (Supp. Table 1). Expression of these genes is enriched in the development of the primitive streak and posterior body formation, including the core gene regulation network for inducing the mesoderm and definitive endoderm such as Tbxt, Wnt3a, and Msgn1. While these genes and their relationships have been studied, the exact genetic changes that drove the evolution of tail-loss in hominoids remain unknown, preventing an understanding of how tail loss affected other human evolutionary events, such as bipedalism.

\section{Results}

\section{A hominoid-specific intronic AluY element in TBXT}

We screened through the 31 human genes - and their primate orthologs - involved in tail 
hominoid genomes and its closest sister lineage, the Old World monkeys (Cercopithecidae).

2 However, we failed to detect candidate variants in hominoid coding sequences that might provide a genetic mechanism for tail-loss evolution (Supp. File 1). We next queried for

4 hominoid-specific genomic rearrangements in the non-coding regions of genes related to tail development. Surprisingly, we found a hominoid-specific Alu element inserted in intron 6 of

$6 T B X T$ (Fig. 1b and Supp. File 1) ${ }^{10,11}$. TBXT codes a highly-conserved transcription factor critical for mesoderm and definitive endoderm formation during embryonic development ${ }^{12,23-25}$.

8 Heterozygous mutations in the coding regions of the TBXT orthologs in tailed animals, such as mouse ${ }^{10}$, Manx $\operatorname{cat}^{26}, \operatorname{dog}^{27}$ and zebrafish ${ }^{28}$, lead to the absence or reduced form of the tail, and homozygous mutants are typically not viable. Moreover, this particular Alu insertion is from the AluY subfamily, a relatively 'young' but not human-specific subfamily shared between the genomes of hominoids and Old World monkeys, the activity of which coincides with the evolutionary time when early hominoids lost their tails ${ }^{29}$.

The AluY element in TBXT is not inserted in the vicinity of a splice site; rather, it is $>500 \mathrm{bp}$ from exon 6 of TBXT, the nearest coding exon. As such, it would not be expected to lead to an alternative splicing event, as found for other intronic Alu elements affecting splicing ${ }^{30-32}$. However, we noted the presence of another Alu element (AluSx1) in the reverse orientation in intron 5 of $T B X T$, that is conserved in all simians. Together, the AluY and AluSx1 elements form an inverted repeat pair (Fig. 1b). We thus posited that upon transcription, the simian-specific AluSx1 element pairs with the hominoid-specific AluY element, forming a stem-loop structure in

22 the TBXT pre-mRNA and trapping exon 6 in the loop (Fig. 1c). An inferred RNA secondary structure model supported an interaction between these two Alu elements ${ }^{33}$ (Fig. S1). The

24 secondary structure of the transcript may thus conjoin the splice donor and receptor of exons 5 and 7, respectively, and promote the skipping of exon 6, leading to a hominoid-specific and in26 frame alternative splicing isoform, TBXT- $\triangle$ exon6 (Fig. 1c). Indeed, we validated the existence 
of TBXT- $\triangle$ exon6 transcripts in human and its corresponding absence in mouse, which lacks the

2 Alu elements, using an embryonic stem cells (ESCs) in vitro differentiation system that induces TBXT expression similar to that present in the primitive streak of the embryo (Fig. S2) ${ }^{34,35}$.

4 Considering the high conservation of TBXT exon 6 and its potential transcriptional regulation function (Fig. S3), we thus hypothesized that in humans and apes, the TBXT- $\Delta$ exon6 isoform

6 protein disrupts tail elongation during embryonic development, leading to the reduction or loss of an external tail (Fig. 1c).

a
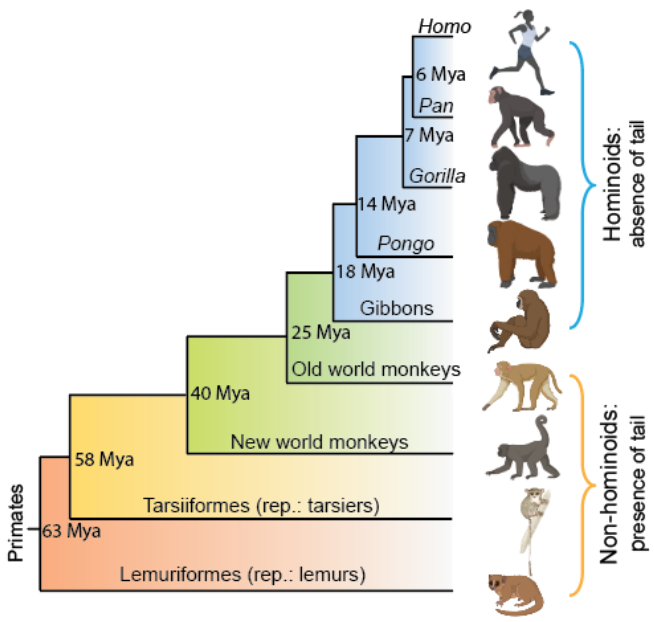

c

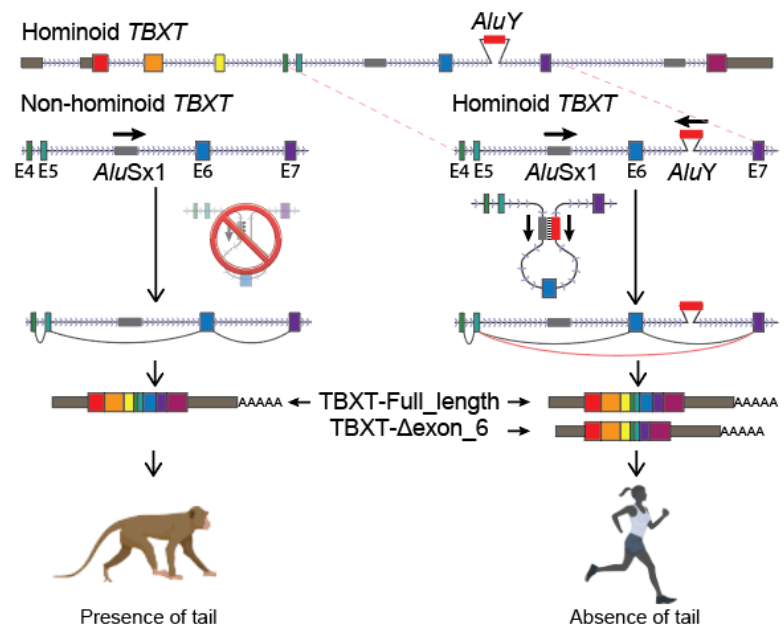

b

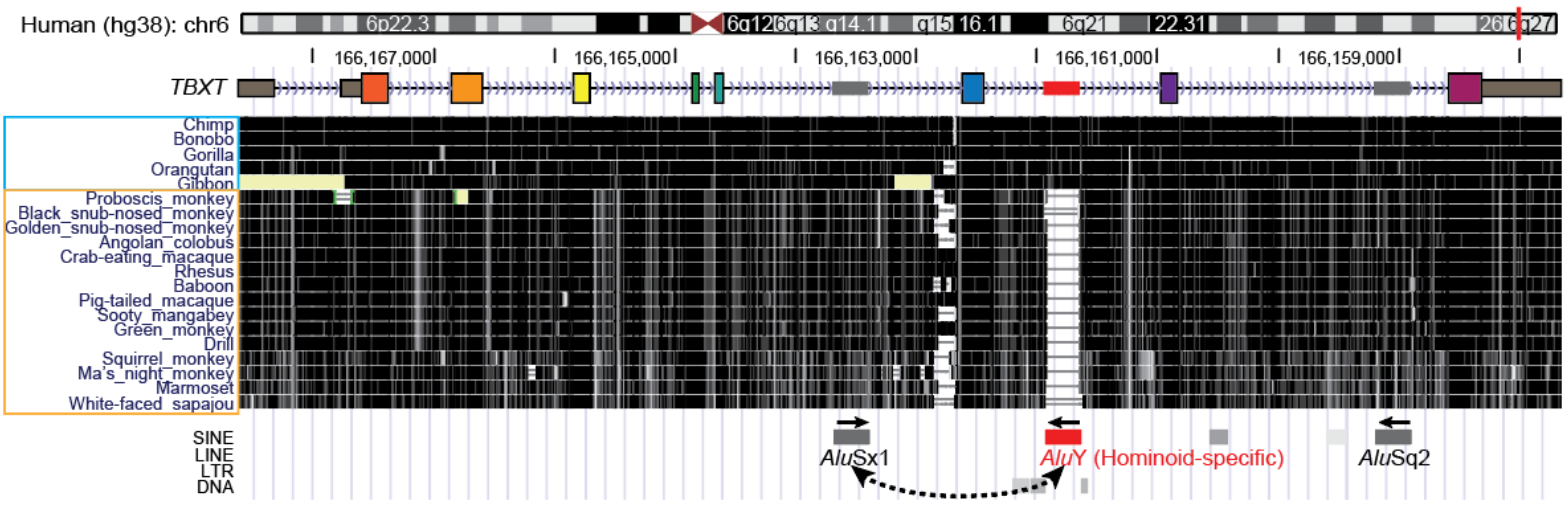

Fig. 1 | Evolution of tail loss in hominoids. a, Tail phenotypes across the primate phylogenetic tree. $\mathbf{b}$,

UCSC Genome browser view of the conservation score through multi-species alignment at the TBXT locus across primate genomes ${ }^{36}$. The hominoid-specific AluY element is labelled in red. c, Schematic of the hypothesized mechanism of tail-loss evolution in hominoids. 


\section{AluY insertion in TBXT induces alternative splicing, and requires interaction with AluSx1}

2 To test whether both $A l u Y$ and $A / u S x 1$ are required to induce the hominoid-specific alternatively spliced isoform of TBXT, we used CRISPR/Cas9 in human ESCs to individually delete the

4 hominoid-specific AluY element and - in a separate line - its potentially interacting counterpart AluSx1 (Fig. 2a, S4a). Again, we adapted the hESC in vitro differentiation system to mimic the

6 TBXT expression in the embryo (Fig. S2) ${ }^{34}$. We found that deleting the AluY almost completely eliminated the generation of the TBXT- $\triangle$ exon6 isoform transcript (Fig. $\mathbf{2 b}$, middle). Similarly,

8 deleting the interacting partner, AluSx1, sufficed to repress this alternatively spliced isoform (Fig. 2b, right). These results support the notion that the hominoid-specific AluY insertion induces a novel TBXT- $\triangle$ exon6 AS isoform, through an interaction with the neighboring AluSx1 element (Fig. 2c, top).

Interestingly, we found that wild-type differentiated hESCs also express a minor, previously unannotated transcript that excludes both exon 6 and exon 7, leading to a frameshift and early truncation at the protein level (Fig. 2b, left, and S4b). Whereas deleting AluY slightly enhanced the abundance of this TBXT- $\triangle$ exon6\&7 transcript, deleting AluSx1 in intron 5 completely eliminated this transcript (Fig. 2b). This may be best explained by a secondary interaction of the AluSx1 element with a distal AluSq2 element in intron 7. In this scenario, the secondary interaction would occur at a lower probability than the AluY-AluSx1 interaction pair (Fig. 2c, bottom). These results further support an interaction among intronic transposable elements affecting splicing of the conserved TBXT regulator (Fig. 2c, S4b). 


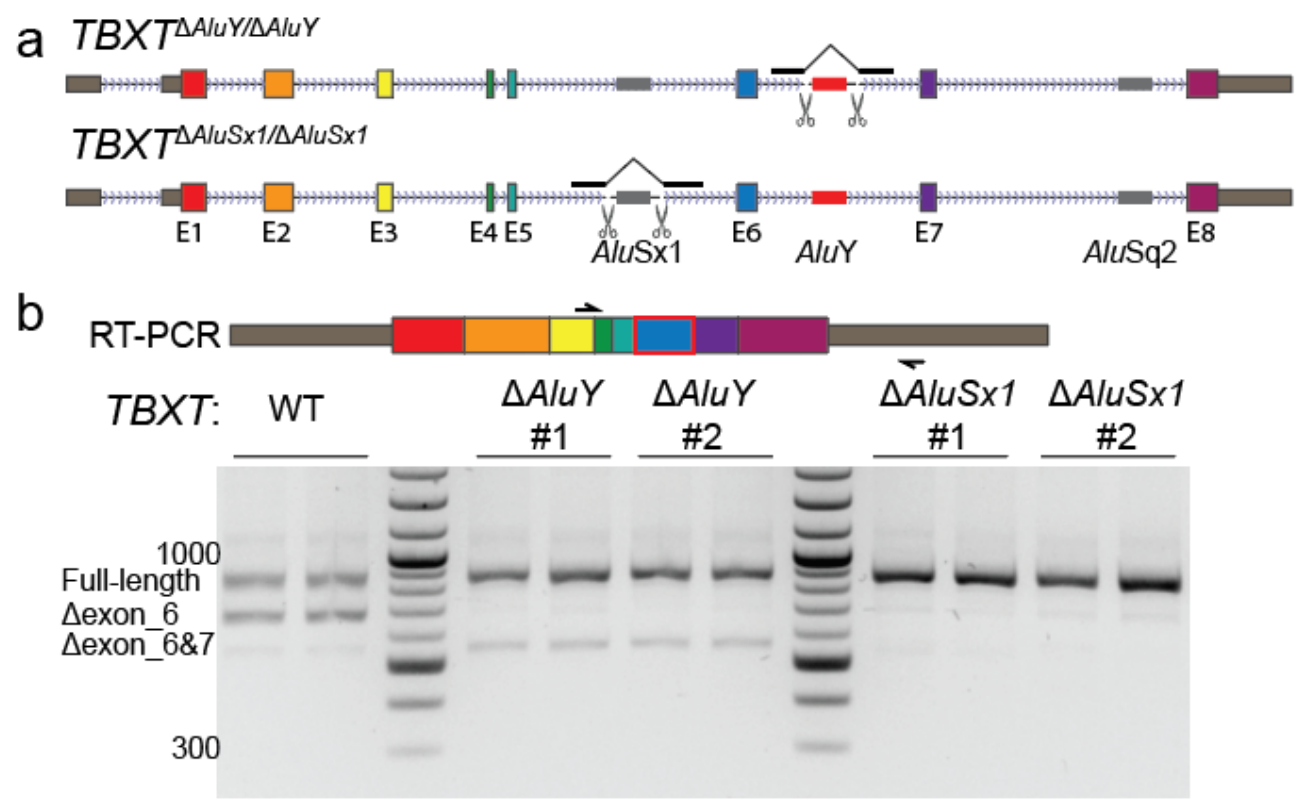

C

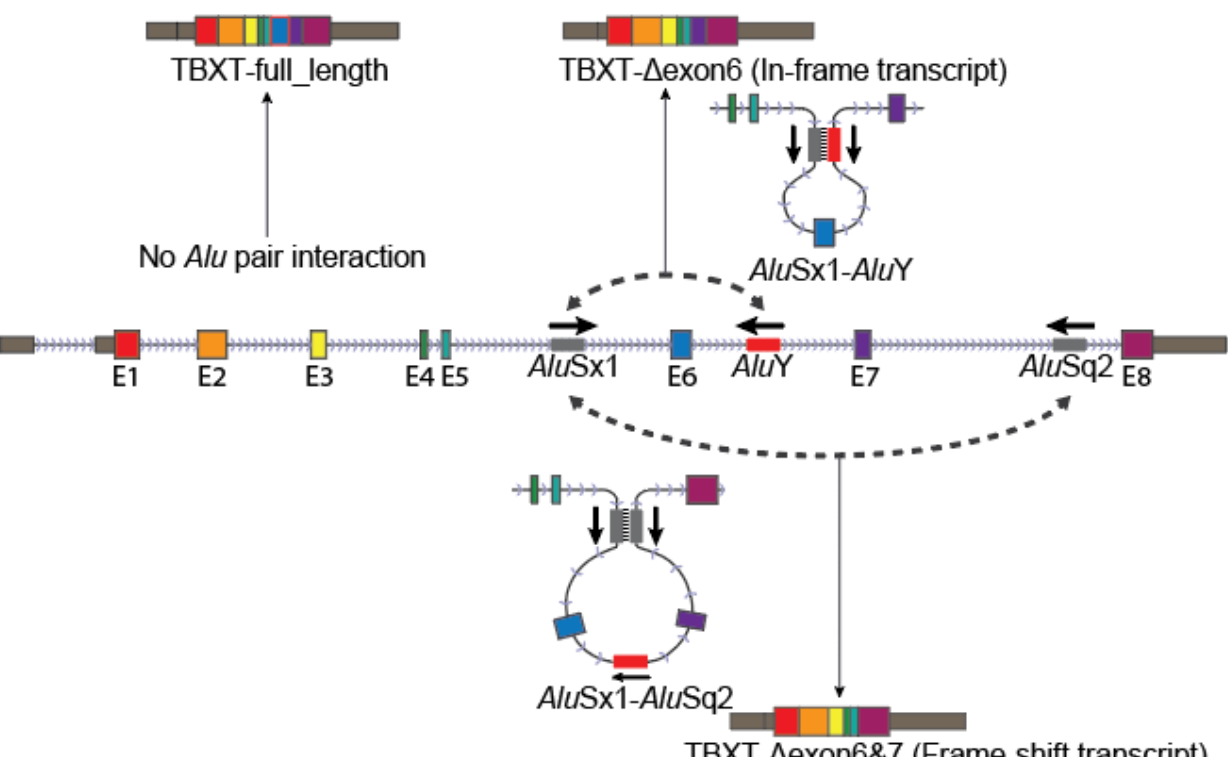

TBXT- $\triangle$ exon6\&7 (Frame-shift transcript)

2 Fig. 2 | Both AluY and AluSx1 are required for TBXT alternative splicing. a, CRISPR-generated homozygous knock-outs of the AluY element in intron 6 and (in a separate line) AluSx1 element in intron

45 of TBXT. b, RT-PCR results of TBXT transcripts isolated from differentiated hESCs of wild-type, $\triangle A / u Y$, and $\triangle A l u S \times 1$ genotypes. Each genotype ( $\triangle A l u Y$ and $\triangle A l u S \times 1)$ was analyzed by two independent

6 replicate clones. c, A schematic of Alu interactions and the corresponding TBXT transcripts in human,

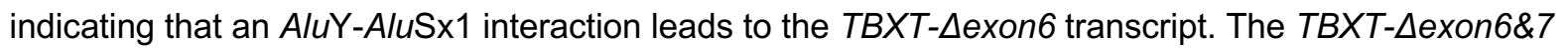
8 transcript may stem from an AluSx1-AluSq2 interaction. 


\section{Tbxt-Dexon6 is sufficient to induce tail loss in mice}

2 To test whether the TBXT- $\triangle$ exon6 isoform is sufficient to induce tail loss, we generated a heterozygous mouse $T b x t^{\Delta e x o n 6 /+}$ model (Fig. 3a). TBXT is highly conserved in vertebrates and

4 human and mouse protein sequences share $91 \%$ identity with a similar exon/intron architecture $^{11}$. We could thus simulate a TBXT- $\Delta$ exon6 isoform by deleting exon 6 in mice,

6 forcing splicing of exons 5 with exon 7. The $T b x t^{\Delta e x o n 6 /+}$ heterozygous mouse thus mimics the TBXT gene in human, which expresses both full-length and $\triangle$ exon6 splice isoforms (Fig. 2b and $3 b-c)$.

Studying the phenotypes of the $T b x t^{\Delta e x o n 6 /+}$ mice, we found that simultaneous expression of both isoforms led to strong but heterogeneous tail morphologies, including no-tail and short-tail

12 phenotypes (Fig. 3d-e, S5). Specifically, 21 of the 63 heterozygous mice showed tail phenotypes, while none of their 35 wild-type littermates showed phenotypes (Table 1). The incomplete penetration of phenotypes among the heterozygotes was stable across generations and founder lines: no-/short-tailed $\left(T b x t^{\Delta e x o n 6 /+}\right)$ parent can give birth to long-tailed $T b x t^{\Delta e x o n 6 /+}$ mice, whereas long-tailed $\left(T b x t^{\Delta e x o n 6 /+}\right)$ parents can give birth to pups with varied tail phenotypes (Table 1, Fig. S5), providing further evidence that the presence of TBXT- $\triangle$ exon6 suffices to induce tail loss.

20 To control for the possibility that zygotic CRISPR targeting induced off-targeting DNA changes at the Tbxt locus, we performed Capture-seq covering the Tbxt locus and $\sim 200 \mathrm{~kb}$ of both upstream and downstream flanking regions ${ }^{37}$ (Fig. S6). Capture-seq did not detect any offtargeting at the Tbxt locus across three independent founder mice, supporting our conclusion

24 that the observed tail phenotype from the $T b x t^{\Delta \text { exon6/+ }}$ mice derived from the $T b x t-\Delta e x o n 6$ isoform. 
2 Table 1. Genotype and phenotype analyses of the Tbxt ${ }^{\Delta e x o n 6 /+}$ intercrossed F2 pups.

\begin{tabular}{|c|c|c|c|c|c|c|c|}
\hline \multirow[b]{2}{*}{ Genotypes } & \multirow{2}{*}{$\begin{array}{l}\text { Total F2 } \\
\text { pups }\end{array}$} & \multirow{2}{*}{$\begin{array}{l}\text { Pups with tail } \\
\text { phenotypes }\end{array}$} & \multicolumn{3}{|c|}{ Tail phenotypes } & \multirow{2}{*}{ 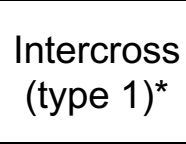 } & \multirow{2}{*}{$\begin{array}{c}\text { Intercross } \\
\text { (type 2) }\end{array}$} \\
\hline & & & $\begin{array}{l}\text { No- } \\
\text { tail }\end{array}$ & $\begin{array}{c}\text { Short- } \\
\text { tail }\end{array}$ & $\begin{array}{c}\text { Kinked- } \\
\text { tail }\end{array}$ & & \\
\hline$T b x t^{\Delta e x o n 6 / \Delta e x o n 6}$ & 0 & 0 & 0 & 0 & 0 & 0 & 0 \\
\hline Tbxt $t^{\Delta e x o n 6 /+}$ & 63 & 21 & 4 & 9 & 8 & $17(7)^{* *}$ & $46(14)$ \\
\hline $\mathrm{Tb} \mathrm{xt}^{+/+}$ & 35 & 0 & 0 & 0 & 0 & $7(0)$ & $28(0)$ \\
\hline
\end{tabular}

Note: *: Type 1 intercrossing: at least one of the parent mice is no-/short-tailed. Type 2 intercrossing: both parent mice are long-tailed.

**: Numbers in parentheses indicate the number of pups with tail phenotypes.

\section{Homozygous removal of Tbxt-Aexon6 is lethal}

10 The human TBXT gene expresses a mixture of TBXT- $\triangle$ exon6 and TBXT-full length transcripts induced as we inferred by the AluY insertion and interaction with AluSx1 - while mouse Tbxt

12 only expresses the full length Tbxt. Thus, we next inquired into the mode by which homozygous TBXT- $\Delta$ exon6 mutation ( $T b x t^{\Delta e x o n 6 / \Delta e x o n 6}$ ) affects development. Intercrossing the $T b x t^{\Delta e x o n 6 /+}$ mice

14 across multiple litters and replicated in different founders, we failed to produce viable homozygotes (Table 1). Dissecting intercrossed embryos at E11.5 showed that homozygotes either arrested development at $\sim \mathrm{E} 9$ or developed with spinal cord malformations that consequently led to death at birth (Fig. S7). We noted that the Tbxt $t^{\Delta e x o n 6 / \Delta e x o n 6}$ embryos showed malformations of the spinal cord similar to spina bifida in humans. Together, while the $\mathrm{Tbxt}^{\wedge \text { exon6/+ }}$ mice present incomplete penetrance of the tail phenotypes requires further

20 investigation, these results indicate that the TBXT- $\triangle$ exon6 isoform, which in human is induced by the intronic AluY-AluSx1 interaction, may indeed be the key driver of tail-loss evolution in 22 hominoids. 


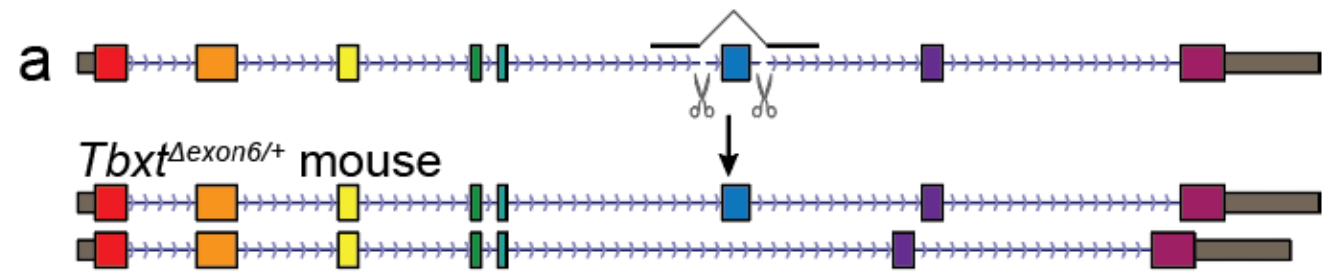

b Tbxt gene transcripts in human and mouse models $\mathrm{Tbxt}^{+/+}$mouse $\quad \mathrm{Tbxt}^{\Delta e x o n 6 /+}$ mouse Human $\left(T B X T^{+/+}\right)$

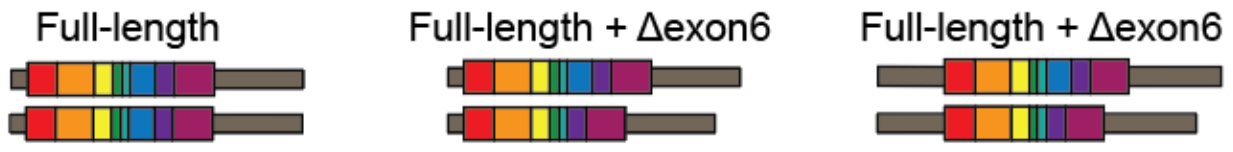

C
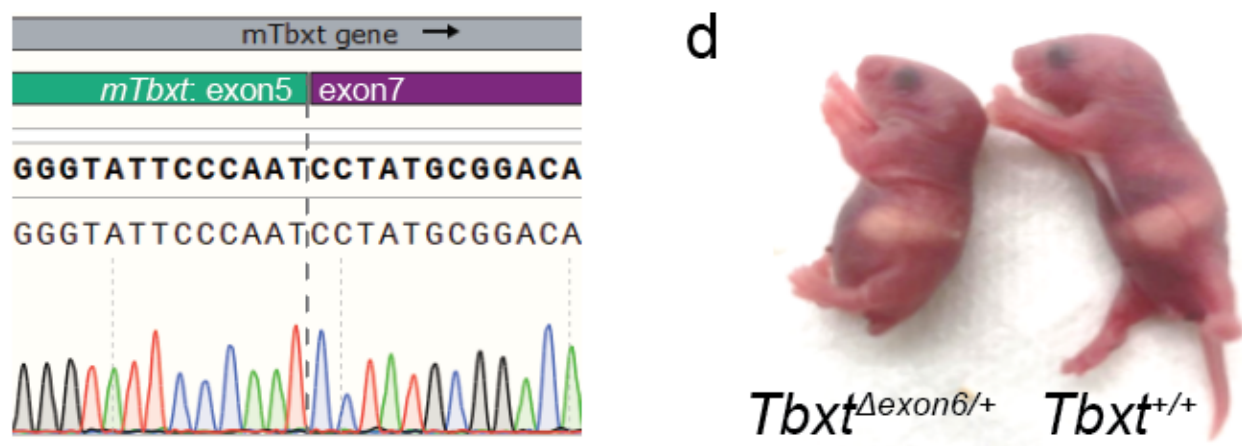

e

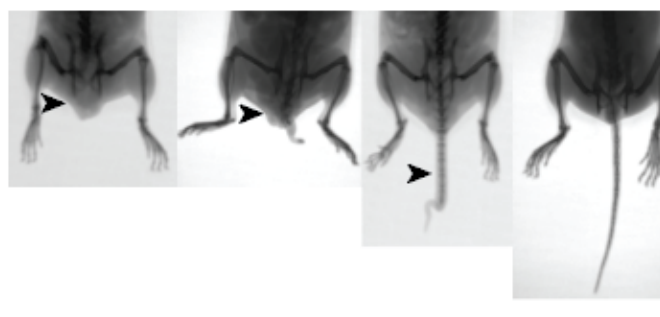

No-tail No-tail Short-tail Short-tail

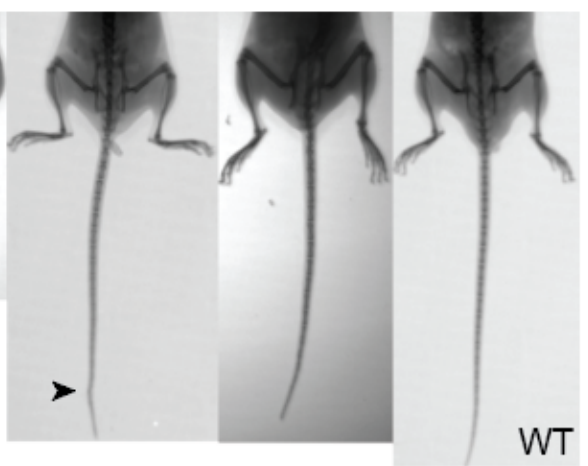

(no sv\&cv) (no cv) $\quad(2-5 \mathrm{cv}) \quad(8-12 \mathrm{cv})$ Kinked-tail Long-tail Normal-tail

Fig. 3 | TBXT-Aexon6 isoform is sufficient to induce tail-loss phenotype. a, CRISPR design for generating a $T b x t^{\Delta e x o n 6 /+}$ heterozygous mouse model. $\mathbf{b}, T_{b x t^{\Delta e x o n 6 /+}}$ mouse mimics TBXT gene expression products in human. c, Sanger-sequencing of the Tbxt RT-PCR product shows that deleting exon 6 in Tbxt leads to correct splicing by fusing exon 5 and 7 . d, A representative Tbxt $t^{\Delta e x o n 6 /+}$ founder mouse (day 1) showing an absence of the tail. Two additional founder mice are shown in Figure S5. e, Tbxt $t^{\Delta e x o n 6 /+}$ heterozygous mice display heterogeneous tail phenotypes varied from absolute no-tail to long-tails. sv, sacral vertebrae; cv, caudal vertebrae. 


\section{Discussion}

2 We have presented evidence that tail-loss evolution in hominoids was driven by the intronic insertion of an AluY element. As opposed to disrupting a splice site, we inferred that this

4 element interacts with a neighboring (simian-shared) AluSx1 element in the neighboring intron, leading to an alternatively spliced isoform which skips an intervening exon (Fig. 1c).

6 Experimental deletion of AluY or its interacting counterpart eliminates such TBXT alternative splicing in differentiated hESCs in the primitive streak state (Fig. 2).

Alternative splicing mediated by Alu-pairing in the TBXT gene demonstrates how an interaction between intronic transposable elements can dramatically modulate gene function to affect a complex trait. The human genome contains $\sim 1.8$ million copies of SINE transposons - including

$12 \sim 1$ million Alus - of which more than $60 \%$ are intronic ${ }^{38}$. Systematically searching for such interactions may lead to the identification of additional functional roles by which these elements

14 impact human development and disease. Interestingly, inverted Alu pairs have been found to contribute to the biogenesis of exonic circular RNAs (circRNA) ${ }^{39}$ through 'backsplicing'. Thus, it

16 is an interesting possibility that the interactions between paired transposable elements may create functional splice variants and circRNA isoforms from the same genetic locus.

We found that expressing the Tbxt- $\Delta$ exon6 transcript - along with the full-length transcript - in

20 mice was sufficient to induce no-tail phenotypes, though with incomplete penetrance (Fig. 3 and

Table 1). It is possible that a heterogeneity of tail phenotypes also existed in the ancestral

22 hominoids upon the initial AluY insertion. Thus, while tail-loss evolution in hominoids may have been initiated by the AluY insertion, additional genetic changes may have then acted to stabilize

24 the no-tail phenotype in early hominoids (Fig. S8). Such a set of genetic events would explain how a change to the AluY in modern hominoids would not result in the re-appearance of the tail. 
2 The specific evolutionary advantage for the loss of the tail is not clear, though it likely involved enhanced locomotion in a non-arboreal lifestyle. We can assume however that the selective

4 advantage must have been very strong since the loss of the tail may have included an evolutionary trade-off of neural tube defects, as demonstrated by the presence of spinal cord

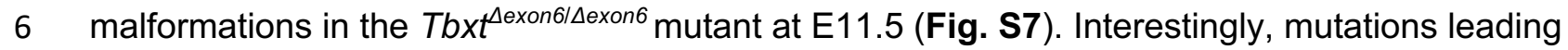
to neural tube defect and/or sacral agenesis have been detected in the coding and noncoding

8 regions of the TBXT gene ${ }^{40-43}$. We thus speculate that the evolutionary tradeoff involving the loss of the tail - made $\sim 25$ million years ago - continues to influence health today. This evolutionary insight into a complex human disease may in the future lead to the design of therapeutic strategies.

\section{Acknowledgments}

We thank Naoya Yamaguchi, Eric Wang, John Shin, Susan Liao, Huiyuan Zhang, and the members of the Yanai and Boeke labs for constructive comments and suggestions. We thank Megan Hogan and Raven Luther for sequencing assistance, and Michael Ceriello and Ahmad Naimi for assistance with the mice work. This work was supported in part by the NHGRI RM1 HG009491 to J.B., and by the NYU Grossman School of Medicine with funding to I.Y. MTM is partially funded by NIH grant R35GM119703. B.X. was partially supported by the NYSTEM predoctoral fellowship (C322560GG).

\section{Author contributions}

24 B.X. conceived the project. B.X., J.D.B. and I.Y designed the experiments with contribution from W.Z. and S.Y.K. B.X. led and conducted most of the experimental and analysis components, 26 with contribution from W.Z., A.W., R.B. and M.P. E.H., R.B. and M.T.M. contributed to the 
capture-seq validation. A.M. and J.S.D. helped with embryo analysis work. J.D.B. and I.Y.

2 supervised the study. B.X. drafted the manuscript. B.X., J.D.B. and I.Y. edited the manuscript with contribution from all authors.

4

\section{Competing interests}

6 J.D.B is a Founder and Director of CDI Labs, Inc., a Founder of and consultant to Neochromosome, Inc, a Founder, SAB member of and consultant to ReOpen Diagnostics, LLC

8 and serves or served on the Scientific Advisory Board of the following: Sangamo Inc., Modern Meadow Inc., Sample6 Inc., Tessera Therapeutics Inc. and the Wyss Institute. The other authors declare no competing interests. 


\section{Methods}

\section{Gene/protein sequence analysis}

Protein sequences were downloaded from NCBI, and analyzed by the MUSCLE algorithm using MEGA X software with default settings ${ }^{44}$. Multiple species gene sequence alignment and analysis were done through the Ensembl Comparative Genomics (release 104) module ${ }^{45}$, using available species of hominoids (human, chimpanzee, bonobo, gorilla, orangutan and gibbon) and Old World monkeys (macaque, crab-eating macaque, pig-tailed macaque, olive baboon, drill, black snub-nosed monkey, golden snub-nosed monkey, Ma's night monkey). The identified candidate gene (TBXT) was then visualized through the UCSC genome $\operatorname{browser}^{36}$ (Figure 1), highlighting the multiple sequence alignment mapping to the human genome (hg38) sequences.

\section{RNA secondary structure prediction}

RNA secondary structure prediction of the TBXT intron5-exon6-intron6 sequence was performed using the ViennaRNA RNAfold Web Services (http://rna.tbi.univie.ac.at/). The algorithm calculates the folding probability using minimum free energy (MFE) matrix with default parameters. In addition, the calculation included the partition function and base pairing probability matrix.

\section{Human ESCs culture and differentiation}

Human ESCs (WA01, also called H1, from WiCell Research Institute) were cultured with StemFlex Medium (Gibco, Cat. No. A3349401) in a feeder cell-free condition. Cells were grown

24 on tissue culture-grade plates coated with hESC-qualified Geltrex (Gibco, Cat. No: A1413302). Geltrex was 1:100 diluted in DMEM/F-12 (Gibco, Cat. No. 11320033) supplemented with 1X 
GlutaMax (100X, Gibco, Cat. No. 35050061) and 1\% Penicillin-Streptomycin (Gibco, Cat. No.

2 15070063). Before seeding hESCs, the plate was treated with Geltrex working solution in a tissue culture incubator $\left(37^{\circ} \mathrm{C}\right.$ and $\left.5 \% \mathrm{CO}_{2}\right)$ for at least $1 \mathrm{~h}$.

Human ESC maintenance and culturing condition were performed according to the manufacturer's protocol of StemFlex Medium. Briefly, StemFlex complete medium was made by

6 combining StemFlex basal medium $(450 \mathrm{~mL})$ with $50 \mathrm{~mL}$ of StemFlex supplement (10X), plus $1 \%$ Penicillin-Streptomycin. Each Geltrex-coated well on a 6-well plate was seeded with 200K cells

8 for $\sim 80 \%$ confluence in 3-4 days. Human ESCs were cryopreserved in PSC Cryomedium (Gibco, Cat. No. A2644601). The culturing medium was supplemented with 1X RevitaCell (100X, Gibco, Cat. No. A2644501, which is also included in the PSC Cryomedium kit) when cells had gone through stressed condition, such as freezing-and-thawing or nucleofection. RevitaCell supplemented medium was replaced with regular StemFlex complete medium on the second day. hESCs grown under RevitaCell condition might become stretched, but would recover after replacing to the normal StemFlex complete medium.

The human ESC differentiation assay to induce a gene expression pattern of primitive streak was adapted from Xi et $a^{\beta 4}$. On day -1 , freshly cultured hESC colonies were dissociated into clumps (2-5 cells) with Versene buffer (with EDTA, Gibco, Cat. No. 15040066). The dissociated cells were seeded on Geltrex-coated 6 -well tissue culture plates at $25,000 \mathrm{cells} / \mathrm{cm}^{2}$ (0.25M per well in the 6-well plates) in StemFlex complete medium. Differentiation to the primitive streak state was initiated on the next day (day 0) by switching StemFlex complete medium to basal differentiation medium. Basal differentiation medium $(50 \mathrm{~mL})$ was made with 48.5mL DMEM/F-12, 1\% GlutaMax (500uL), 1\% ITS-G (500uL, Gibco Cat. No. 41400045), and $1 \%$ penicillin-streptomycin $(500 \mu \mathrm{L})$, and supplemented with $3 \mu \mathrm{M}$ GSK3 inhibitor CHIR99021

$24(10 \mu \mathrm{L}$ of $15 \mathrm{mM}$ stock solution in DMSO. Tocris, Cat. No. 4423). The cells were collected at differentiation day 1 to 3 for downstream experiments, which confirmed the expression 26 fluctuations of mesoderm genes (TBXT and MIXL1) in a 3-day differentiation period (Fig. S3) ${ }^{34}$. 


\section{Mouse ESC culture and differentiation}

Mouse ESCs derived from C57BL/6J strain background were cultured in a feeder cell-

4 free condition. Cells were grown on tissue culture-grade plates coated with mESC-qualified gelatin. Before plating cells, the plastic tissue culture-treated plates were coated with $0.1 \%$

6 gelatin (EMD Millipore ES-006-B) at room temperature for at least 30min, followed by switching to $\mathrm{mESC}$ medium and warming up the medium at $37^{\circ} \mathrm{C}$ and $5 \% \mathrm{CO} 2$ incubator for at least

$830 \mathrm{~min}$.

The feeder cell-free mESC culturing medium, also called '80/20' medium, comprises $80 \%$ 2i medium and $20 \%$ mESC medium by volume. $2 \mathrm{i}$ medium was made from a $1: 1$ mix of Advanced DMEM/F-12 (Gibco, Cat. No. 12634010) and Neurobasal-A (Gibco, Cat. No. 10888022), 1X N-2 supplement (Gibco, Cat. No. 17502048), 1X B-27 supplement (Gibco, Cat. No. 17504044), 1X Glutamax (Gibco, Cat. No. 35050061), 0.1 mM Beta-Mercaptoethanol

14 (Gibco, Cat. No. 31350010), 1000 units/mL LIF (Millipore, Cat. No. ESG1107), 1 M MEK1/2 inhibitor (Stemgent, Cat. No. PD0325901), and 3 $\mu$ M GSK3 inhibitor CHIR99021 (Tocris, Cat. No. 4423). mESC medium was made from Knockout DMEM (Gibco, Cat. No. 10829018), containing 15\% Fetal Bovine Serum (GeminiBio, Cat. No. 100-106), $0.1 \mathrm{mM}$ BetaMercaptoethanol, 1X MEM Non Essential Amino Acids (Gibco, Cat. No. 11140050), 1X Glutamax, 1X Nucleosides (Millipore, Cat. No. ES-008-D) and 1000 units/mL LIF. mESC differentiation for inducing Tbxt gene expression was adapted from Pour et al in a feeder cell-free condition ${ }^{46}$. Cells were first plated in $80 / 20$ medium for 24 hours on a gelatin-

22 coated 6-well plate, followed by switching to N2/B27 medium without LIF or 2i for another 2-day culturing. The N2/B27 medium $(50 \mathrm{~mL})$ is made with $18 \mathrm{~mL}$ Advanced DMEM/F-12, $18 \mathrm{~mL}$

24 Neurobasal-A, 9mL Knockout DMEM, 2.5mL Knockout Serum Replacement (Gibco, Cat. No. 10828028), 0.5mL N-2 supplement, $1 \mathrm{~mL}$ B-27 supplement, $0.5 \mathrm{~mL}$ Glutamax (100X), 0.5mL 
supplemented with 34M GSK3 inhibitor CHIR99021 for induced differentiation (day 0). The cells

2 were collected at differentiation day 1 to 3 for downstream experiments, which showed consistent results of Tbxt gene expression fluctuations in a 3-day differentiation period.

\section{CRISPR targeting}

All guide RNAs of the CRISPR experiments were designed using CRISPOR algorithm through its predicted target sites integrated in the UCSC genome browser ${ }^{47}$. Guide RNAs were cloned into the pX459V2.0-HypaCas9 plasmid (AddGene plasmid \#62988), or its custom derivative by replacing the puromycin resistance gene to blasticidin resistance gene. Guide

RNAs in this study were designed in pairs to delete the intervening sequences. The sequence and targeting sites of the guide RNAs were listed below:

\begin{tabular}{|l|l|l|l|}
\hline & PAM & Guide RNA sequence & Locus (hg38/mm10) \\
\hline Delete AluY & TGG & AGACTGTGCCCACTCTCGGG & chr6:166161988-166162010 \\
\cline { 2 - 4 } & TGG & GATAGACCATAAAGATCCCC & chr6:166161557-166161579 \\
\hline \multirow{2}{*}{ Delete AluSx1 } & GGG & CACAGTAGTTGTCCCGCTAG & chr6:166163730-166163752 \\
\cline { 2 - 4 } & AGG & GAATGGGGGGAGCTTAAACC & chr6:166163290-166163312 \\
\hline $\begin{array}{l}\text { Delete Tbxt- } \\
\text { exon6 }\end{array}$ & TGG & ATTTCGGTTCTGCAGACCGG & chr17:8438362-8438384 \\
\cline { 2 - 4 } & GGG & CAAGATGCTGGTTGAACCAG & chr17:8438920-8438942 \\
\hline
\end{tabular}

12

All oligos (plus Goldern-Gate assembly overhangs) were synthesized from Integrated DNA Technologies (IDT) and ligated into empty pX459V2.0 vector following standard Golden Gate Assembly protocol using Bbsl restriction enzyme (NEB, Cat. No. R3539). The constructed plasmids were purified from 3mL E. coli cultures using ZR Plasmid MiniPrep Purification Kit (Zymo Research, Cat. No. D4015) for sequence verification. Plasmids delivered into ESCs were purified from 250mL E. coli cultures using PureLink HiPure Plasmid Midiprep Kit (Invitrogen, Cat. No. K210005). To facilitate DNA delivery to ESCs through nucleofection, the purified plasmids were resolved in Tris-EDTA buffer $(\mathrm{pH} 7.5)$ for a concentration of at least $1 \mu \mathrm{g} / \mu \mathrm{L}$ in a sterile hood. 


\section{DNA delivery}

DNA delivery into human/mouse ESCs for CRISPR/Cas9 targeting were performed

4 using a Nucleofector 2b Device (Lonza, Cat. No. BioAAB-1001). Human Stem Cell Nucleofector Kit 1 (Cat. No. VPH-5012) and mESC Nucleofector Kit (Lonza, Cat. No. VVPH-1001) were used

6 for delivering DNA into human and mouse ESCs, respectively. ESCs were double-feeded the day before the nucleofection experiment to maintain a superior condition.

Before performing nucleofection on human ESCs, $6 \mathrm{~cm}$ tissue culture plates were treated with $0.5 \mu \mathrm{g} / \mathrm{cm}^{2}$ rLaminin-521 in a $37^{\circ} \mathrm{C}$ and $5 \% \mathrm{CO}_{2}$ incubator for at least $2 \mathrm{~h}$. rLaminin-521treated plates give better viability when seeding hESCs as single cells. Cultured human ESCs were then washed with PBS, and dissociated into single cells using TrypLE Select Enzyme (no

12 phenol red. Gibco, Cat. No. 12563011). One million hES single cells were nucleofected using program A-023 according to the manufacturer's instruction of the Nucleofector $2 b$ device.

14 Transfected cells were transferred on the rLaminin-521-treated $6 \mathrm{~cm}$ plates with pre-warmed StemFlex complete medium supplementing with $1 \mathrm{X}$ RevitaCell but not Penicillin-Streptomycin. Antibiotic selection was performed $24 \mathrm{~h}$ after nucleofection with puromycin (Gibco, Cat. No. A1113802).

Mouse ESCs were dissociated into single cells using StemPro Accutase (Gibco, Cat. No. A1110501) and five million cells were transfected using program A-023 according to the manufacturer's instruction. Cells were plated onto gelatin-treated $10 \mathrm{~cm}$ plates, followed by antibiotic selection 24h after nucleofection with blasticidin (Gibco, Cat. No. A1113903). Together with the pX459V2.0-HypaCas9-gRNA plasmids for nucleofection, a singlestrand DNA oligo were co-delivered for micro homology-induced deletion of the targeted sites $^{48}$.

24 These ssDNA sequences were synthesized from IDT through its Ultramer DNA Oligo service, including phosphorothioate bond modification on the three bases of each end. Detailed sequence information was listed below (“|” indicates a junction site): 


\begin{tabular}{|c|c|}
\hline Delete $T B X T$-AluY & 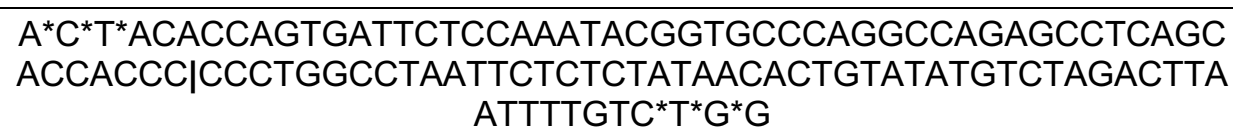 \\
\hline $\begin{array}{l}\text { Dele } \\
\text { AluS }\end{array}$ & $\begin{array}{l}\text { C*A*G*TGCTGCCTGGAGAATTGTTAGTAGTTTGGAAATTGAAGCCACAGTAGTTGT } \\
\text { CCCGC|ACCAGGAGAGTGAGCAGTAAAAGGGTCTACCCCCAGCTAGGAAGGCACCT } \\
\text { CCCGTC* }{ }^{*} \mathrm{C}^{*} \mathrm{~T}\end{array}$ \\
\hline Delete & $\begin{array}{l}\text { T*T*T*ATTCTAGAGCCCATTAACATATCACTCCTGCTCACTTGGTAGAAAGCCACCG| } \\
\text { CAGGGGTCCCCAAGGAGGCTTTCATTTCAATATCCATGTGCCTCAGAACATG*C*C* } \\
\text { C }\end{array}$ \\
\hline
\end{tabular}
standard protocol. The genotyping primers were listed below:

\begin{tabular}{|c|c|c|}
\hline Target site & Orientation & Sequence \\
\hline \multirow{2}{*}{$\begin{array}{l}\text { Delete } T B X T-A l u Y: \\
\text { read through }\end{array}$} & $\mathrm{F}$ & CAGCCAGGCTCAAGAATTCC \\
\hline & $\mathrm{R}$ & GACTTCCTAACCCAATAAGGTCC \\
\hline \multirow{2}{*}{$\begin{array}{l}\text { Delete } T B X T-A l u Y \text { : left } \\
\text { junction }\end{array}$} & $\mathrm{F}$ & CAGCCAGGCTCAAGAATTCC \\
\hline & $\mathrm{R}$ & GTGTTCCTAATATTGGAGCATGC \\
\hline \multirow{2}{*}{$\begin{array}{l}\text { Delete } T B X T-A l u S \times 1: \\
\text { read through }\end{array}$} & $\mathrm{F}$ & TCCTAGGCTGATTGAACAACCAG \\
\hline & $\mathrm{R}$ & CAAGGCAGGTGAGCTTTCC \\
\hline \multirow{2}{*}{$\begin{array}{c}\text { Delete TBXT-AluSx1: } \\
\text { left junction }\end{array}$} & $\mathrm{F}$ & TCCTAGGCTGATTGAACAACCAG \\
\hline & $\mathrm{R}$ & TTAAGCTCCCCCCATTC \\
\hline \multirow{2}{*}{$\begin{array}{l}\text { Delete } T b x t-e x o n 6: \\
\text { read through }\end{array}$} & $\mathrm{F}$ & GCAGTCTGAGTCCTACCTGTG \\
\hline & $\mathrm{R}$ & TGTCAGTCTGGTTCTACACCTGGAGAGTCTTTGATC \\
\hline \multirow{2}{*}{$\begin{array}{l}\text { Delete } T b x t \text {-exon6: left } \\
\text { junction }\end{array}$} & $\mathrm{F}$ & GCAGTCTGAGTCCTACCTGTG \\
\hline & $\mathrm{R}$ & GTACAGGACCTACTTGGAGAGC \\
\hline \multirow{2}{*}{$\begin{array}{l}\text { Delete Tbxt-exon6: } \\
\text { right junction }\end{array}$} & $\mathrm{F}$ & GACAGGACTGAGTCTCAAGC \\
\hline & $\mathrm{R}$ & TGTCAGTCTGGTTCTACACCTGGAGAGTCTTTGATC \\
\hline
\end{tabular}

4

\section{Splicing isoforms detection}

Total RNAa were collected from the undifferentiated or differentiated cells of both human and mouse ESCs, using standard column-based purification kit (QIAGEN RNeasy Kit, Cat. No. 74004). DNase treatment was applied during the purification to remove any potential DNA contamination. Following extraction, RNA quality was checked through electrophoresis based on the ribosomal RNA integrity. Reverse transcription was performed with $1 \mu \mathrm{g}$ of high-quality total RNA for each sample, using High-Capacity RNA-to-cDNA ${ }^{\mathrm{TM}}$ Kit (Applied Biosystems, Cat. No. 4387406). DNA oligos used for PCR/RT-PCR/RT-qPCR were listed below: 


\begin{tabular}{|c|c|c|}
\hline Target site & Orientation & Sequence \\
\hline \multirow{2}{*}{$\begin{array}{c}\text { Human TBXT: exon3-8 } \\
\text { (Fig. 2B) }\end{array}$} & $\mathrm{F}$ & GGTGACTGCTTATCAGAACGAGGAG \\
\hline & $\mathrm{R}$ & TACTGAGGCTGCATTTCCTTCTTAACC \\
\hline \multirow{2}{*}{ Human TBXT (Fig. S2) } & $\mathrm{F}$ & CCTCATAGCCTCATGGACACCTG \\
\hline & $\mathrm{R}$ & TCTTAACCTGAGACTGCCACTGG \\
\hline \multirow{2}{*}{$\begin{array}{l}\text { Human MIXL1 (Fig. } \\
\text { S2) }\end{array}$} & $\mathrm{F}$ & GGCGTCAGAGTGGGAAATCC \\
\hline & $\mathrm{R}$ & GGCAGGCAGTTCACATCTACC \\
\hline \multirow{2}{*}{$\begin{array}{l}\text { Human ACTB1 (Fig. } \\
\text { S2) }\end{array}$} & $\mathrm{F}$ & CACCATTGGCAATGAGCGGTTC \\
\hline & $\mathrm{R}$ & AGGTCTTTGCGGATGTCCACGT \\
\hline \multirow{2}{*}{$\begin{array}{c}\text { Human TBXT: exon4-7 } \\
\text { (Fig. S2) }\end{array}$} & $\mathrm{F}$ & CAGAACGAGGAGATCACAGCTC \\
\hline & $\mathrm{R}$ & GGTACTGACTGGAGCTGGTAGG \\
\hline \multirow{2}{*}{$\begin{array}{c}\text { Mouse Tbxt: exon4-7 } \\
\text { (Fig. S2) }\end{array}$} & $\mathrm{F}$ & CCAGAATGAGGAGATTACAGCCCT \\
\hline & $\mathrm{R}$ & GGATACTGGCTAGAGCCAGTAGG \\
\hline \multirow{2}{*}{ Mouse Actb1 (Fig. S2) } & $\mathrm{F}$ & CATTGCTGACAGGATGCAGAAGG \\
\hline & $\mathrm{R}$ & TGCTGGAAGGTGGACAGTGAGG \\
\hline
\end{tabular}

\section{Mouse work}

All mouse work was done following NYULH's animal protocol guidelines. The Tbxt ${ }^{\text {exon6/+ }}$ heterozygous mouse model was generated through zygotic microinjection, using an experimental protocol adapted from Yang et $a{ }^{49}$. Briefly, Cas9 mRNA (MilliporeSigma, Cat. No. CAS9MRNA), synthetic guide RNAs, and single-stranded DNA oligo were co-injected into the 1-

8 cell stage zygotes following the described procedures ${ }^{49}$. Synthetic guide RNAs were ordered from Synthego as their custom CRISPRevolution sgRNA EZ Kit, with the same targeting sites as used in the CRISPR deletion experiment of mouse ESCs (AUUUCGGUUCUGCAGACCGG and CAAGAUGCUGGUUGAACCAG). The co-injected single-stranded DNA oligo is the same as above mentioned as well. Processed embryos were then in vitro cultured to the blastomeric stage, followed by embryo transferring to the pseudopregnant foster mothers. Following zygotic microinjection and transferring, founder pups were screened based on their abnormal tail phenotypes. DNA samples were collected through ear punches at day $\sim 21$ for genotyping. Upon confirming the heterozygous genotype $\left(T b x t^{\Delta e x o n 6 /+}\right)$, founder mice were backcrossed with wild-type C57B/6J mice for generating heterozygous F1 pups. Due to the 
varied tail phenotypes, intercrossing between F1 heterozygotes were performed in two

2 categories: type1 intercrossing includes at least one parent being no-/short-tailed, whereas type 2 intercrossing were mated between two long-tailed F1 heterozygotes. Both types of

4 intercrossing produced heterogeneous tail phenotypes in F2 $\mathrm{Tbxt}^{\text {Aexon6/+ }}$ pups, confirming the

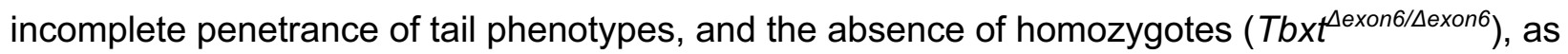

6 summarized in Table 1. To confirm the embryonic phenotypes in homozygotes, embryos were dissected at E11.5 gestation stage from the timed pregnant mice through the standard protocol.

8 Adult mice (>12 weeks) were anesthetized for X-ray imaging of vertebra using a Bruker In-Vivo Xtreme IVIS imaging system.

\section{Capture-seq genotyping}

Capture-seq, or targeted sequencing of the loci of interest, was performed as previously described $^{37}$. Conceptually, capture-seq uses custom biotinylated probes to pull down the genomic loci of interest from the standard whole-genome sequencing libraries, thus enabling sequencing of the specific genomic loci in a much higher depth while reducing the cost.

Genomic DNA were purified from mESCs or ear punches of founder mice using Zymo Quick-DNA Miniprep Plus Kit (Cat. No. D4068) according to manufacturer's instruction. DNA sequencing libraries compatible for Illumina sequencers were prepared following standard protocol. Briefly, $1 \mu \mathrm{g}$ of gDNA was sheared to $500-900$ base pairs in a 96 -well microplate using the Covaris LE220 (450 W, 10\% Duty Factor, 200 cycles per burst, and 90-s treatment time),

22 followed by purification with a DNA Clean and Concentrate-5 Kit (Zymo Research, Cat. No. D4013). Sheared and purified DNA were then treated with end repair enzyme mix (T4 DNA 24 polymerase, Klenow DNA polymerase, and T4 polynucleotide kinase, NEB, Cat. No. M0203, M0210 and M0201, respectively), and A-tailed using Klenow 3'-5'exo- enzyme (NEB, Cat. No. 
M0212). Illumina sequencing library adapters were subsequently ligated to DNA ends, followed

2 by PCR amplification with KAPA 2X Hi-Fi Hotstart Readymix (Roche, Cat. No. KR0370).

Custom biotinylated probes were prepared as bait through nick translation, using BAC

4 DNA and/or plasmids as the template. The probes were prepared to comprehensively cover the whole locus. We used BAC lines RP24-88H3 and RP23-159G7, purchased from BACPAC

6 Genomics, to generate bait probes covering mouse Tbxt locus and $\sim 200 \mathrm{~kb}$ flanking sequences in both upstream and downstream regions. The pooled whole-genome sequencing libraries

8 were hybridized with the biotinylated baits in solution, and purified through streptavidin-coated magnetic beads. Following pull-down, DNA sequencing libraries were quantified with Qubit 3.0

10 Fluorometer (Invitorgen, Cat. No. Q33216) using a dsDNA HS Assay Kit (Invitorgen, Cat. No. Q32851). The sequencing libraries were subsequently sequenced on an Illumina NextSeq 500 sequencer in paired-end mode.

Sequencing results were demultiplexed with Illumina bcl2fastq v2.20 requiring a perfect match to indexing $\mathrm{BC}$ sequences. Low quality reads/bases and Illumina adapters were trimmed with Trimmomatic v0.39. Reads were then mapped to mouse genome (mm10) using bwa visualization in a mirror version of UCSC genome browser. 


\section{Supplementary Figures:}

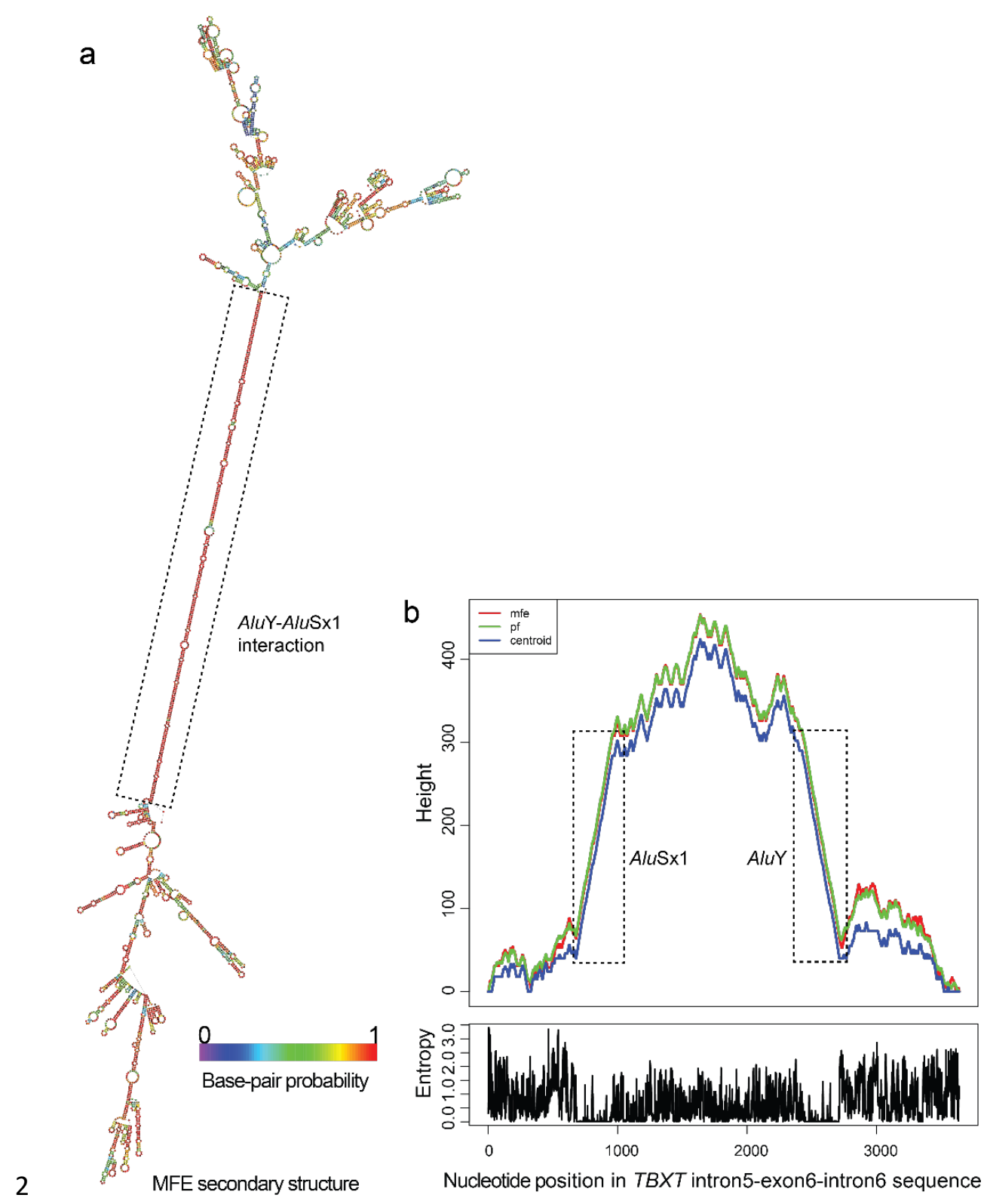

\section{Fig. S1 | RNA structure prediction using RNAfold algorithm of the ViennaRNA package ${ }^{33}$.}

4 a, Predicted RNA secondary structure of the TBXT intron5-exon6-intron6 sequence. The paired AluY-AluSx1 region is highlighted. b, Mountain plot of the RNA secondary structure prediction,

6 showing the 'height' in predicted secondary structure across the nucleotide positions. Height is computed as the number of base pairs enclosing the base at a given position. Overall, the 8 AluSx1 and AluY regions are predicted to form helices with high probability (low entropy). 
a

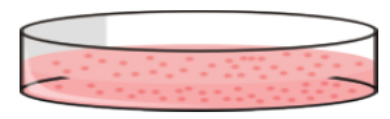

ESCs In vitro Differentiation

GSK3 inhibitor

(CHIR99021)

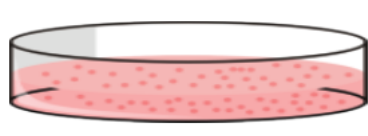

Primitive streak state b

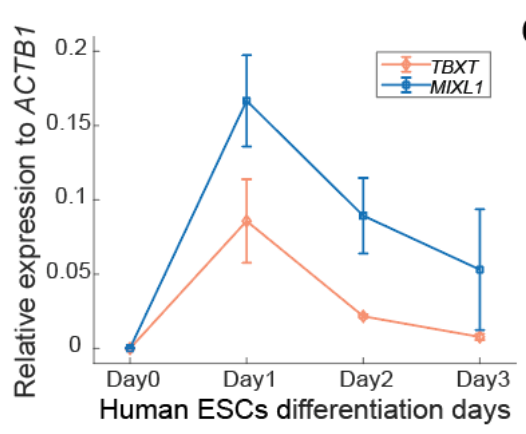

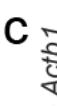

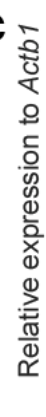
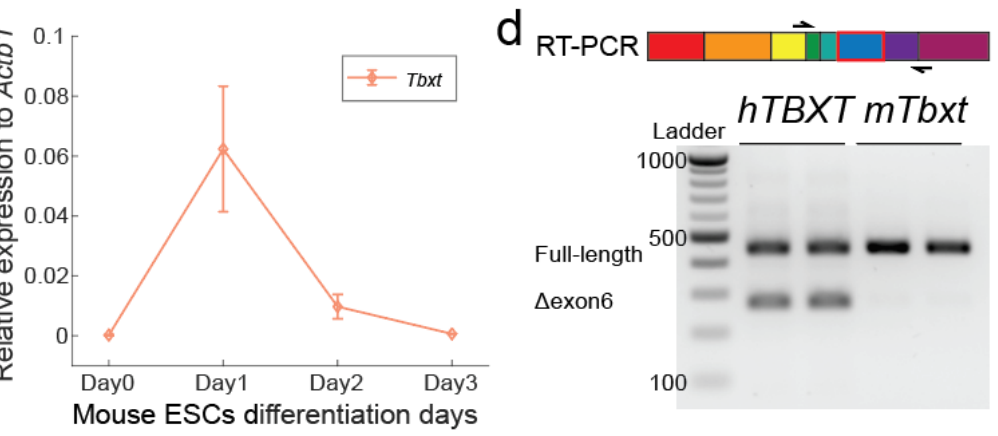

Fig. S2 | Studying TBXT expression isoforms using primitive streak in vitro

differentiation. a, Human and mouse ESCs in vitro differentiation for inducing TBXT

expression. Human and mouse ESCs differentiation assay was adapted from Xi et $\mathrm{a}{ }^{34}$ and Pour et $a{ }^{35}$, respectively. b, Quantitative RT-PCR of TBXT and MIXL1 expression during hESC

6 differentiation, indicating correct induction of mesodermal gene expression program ${ }^{34}$. c, Quantitative RT-PCR of Tbxt expression during mESC differentiation. d, RT-PCR of TBXT/Tbxt

8 transcripts in human and mouse, highlighting a unique $\Delta$ exon6 splicing isoform in human. 
a Homo Sapiens | TBXT- $\triangle$ exon6 Homo Sapiens | TBXT-Full length Pan troglodytes | TBXT Macaca fascicularis | TBXT Macaca mulatta | TBXT Saimiri boliviensis | TBXT Prolemur simus | TBXT Rattus norvegicus | Tbxt Mus musculus | Tbxt Canis lupus familiaris | Tbxt Felis catus | Tbxt

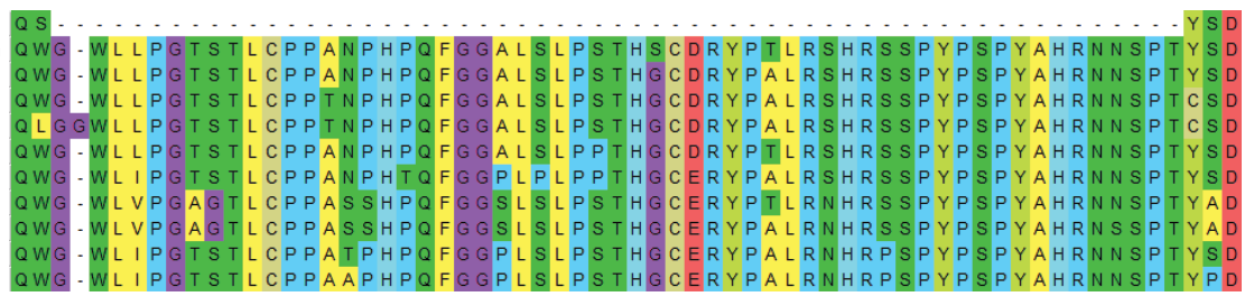

b

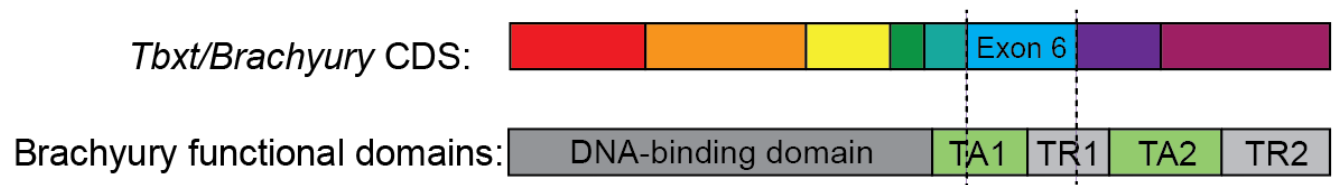

2 Fig. S3 | TBXT exon 6 conservation and functional domain analysis. a, Protein sequence alignment of the TBXT exon 6 region in representative mammals. With the exception of humans 4 and chimpanzees, all are tailed. b, The exon 6-derived peptide of TBXT overlaps with large fractions of transcription regulation domains. TA, transcription activation; TR, transcriptional 6 repression. Functional domain annotation of Brachyury was adapted from Kispert et a/ ${ }^{12}$. 

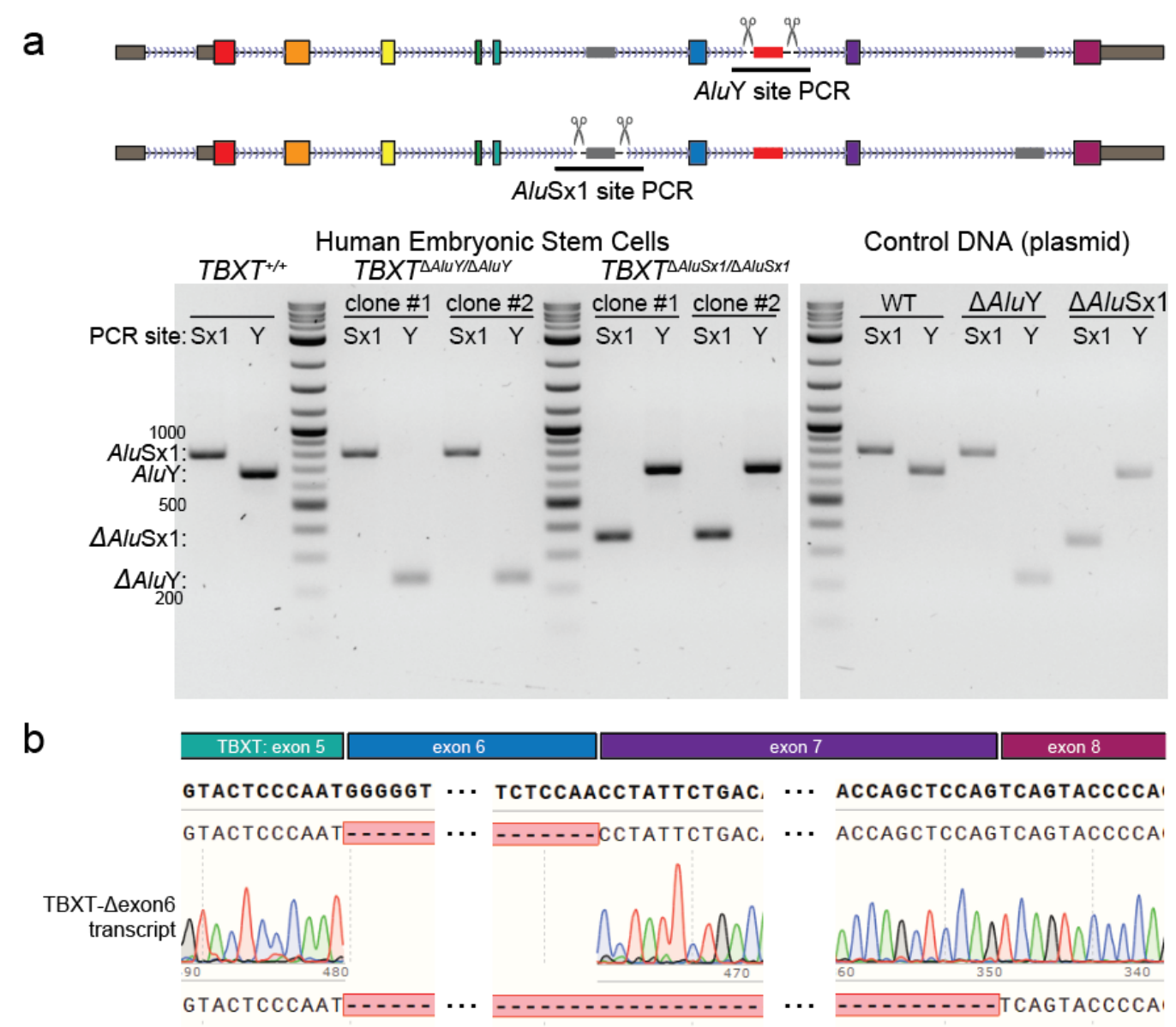

TBXT- $\Delta$ exon6\&7 transcript
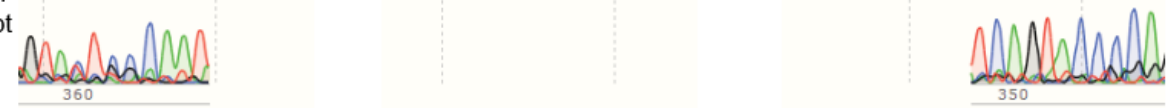

2 Fig. S4 | Validation of hESC CRISPR-deletion clones and the TBXT expression isoforms.

a, PCR validation of the hESC clones with deletions of AluY or AluSx1 in TBXT. PCR validation

4 for each clone or control samples were performed in pairs, each amplifying the AluSx1 locus (Sx1) or the AluY locus $(\mathrm{Y})$, respectively, with primers that bind the two flanking sequences of

6 the deleted region. Each genotype included two independent clones of AluY deletion or AluSx1 deletion, corresponding to the two replicates in Figure 2B. b, Sanger sequencing of the TBXT-

$8 \Delta$ exon6 and TBXT- $\triangle$ exon6\&7 transcripts detected in Figure $2 \mathrm{~B}$. The sequencing results were aligned to the full length TBXT mRNA sequence. 


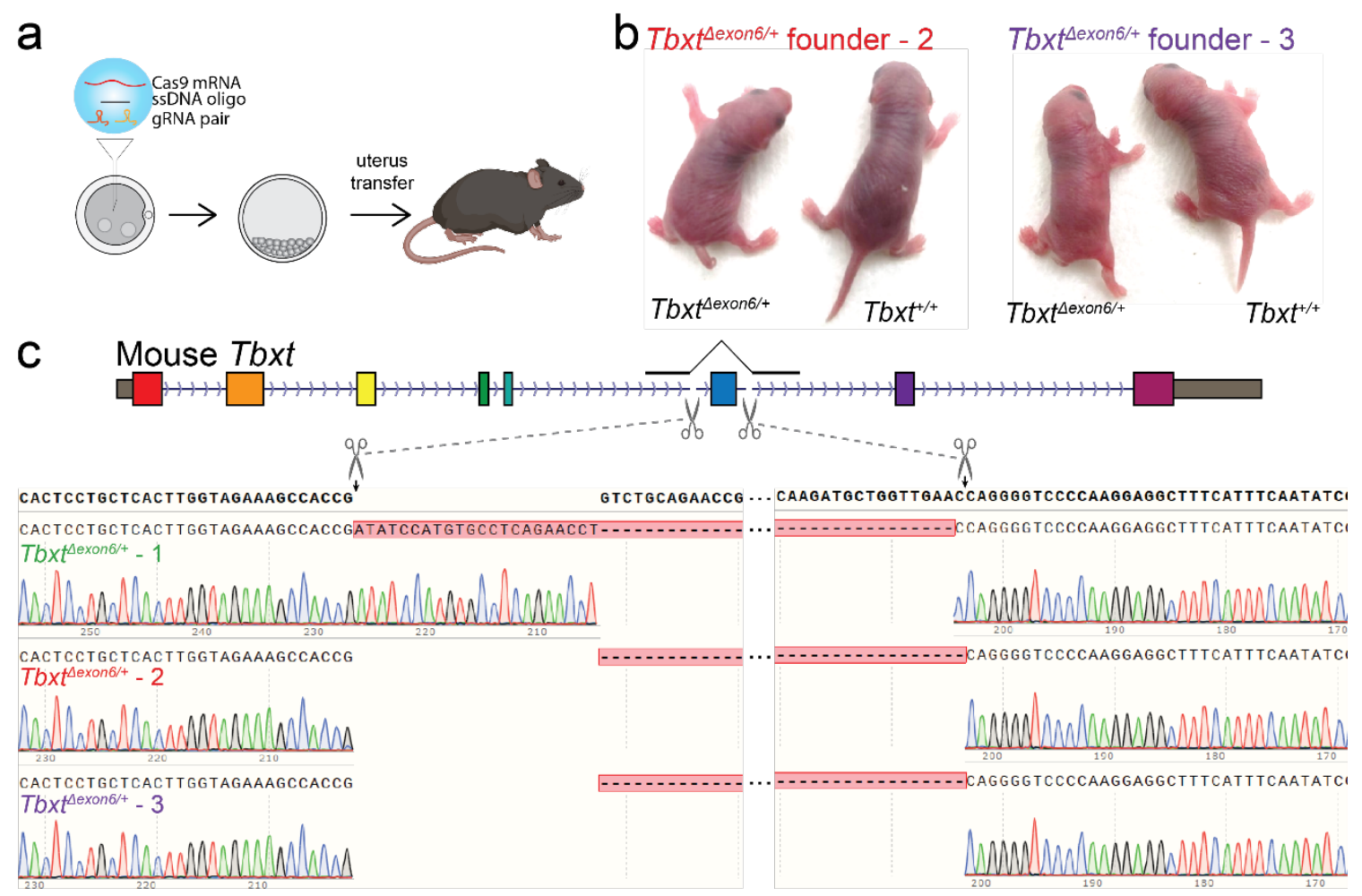

2 Fig. S5 | Tbxt $t^{\text {exon6/+ }}$ founder mice generated through CRISPR/Cas9 targeting in the zygotes. a, Schematic of zygotic injection of CRISPR/Cas9 reactions. b, Two Tbxt ${ }^{\Delta e x o n 6 /+}$

4 founder mice (in addition to the one shown in Fig. 3) indicating an absence or reduced form of the tail (Founders $2 \& 3$ ). c, Sanger sequencing of the exon 6-deleted allele isolated from the 6 genomic DNA of Tbxt $t^{\Delta e x o n 6 /+}$ founder mice. Founder 1 had an unexpected insertion of 23 base pairs at the CRISPR cutting site in the original intron 5 of Tbxt. Both founder 2 and 3 had the 8 exact fusion between the two CRISPR cutting sites in introns 5 and 6 . 


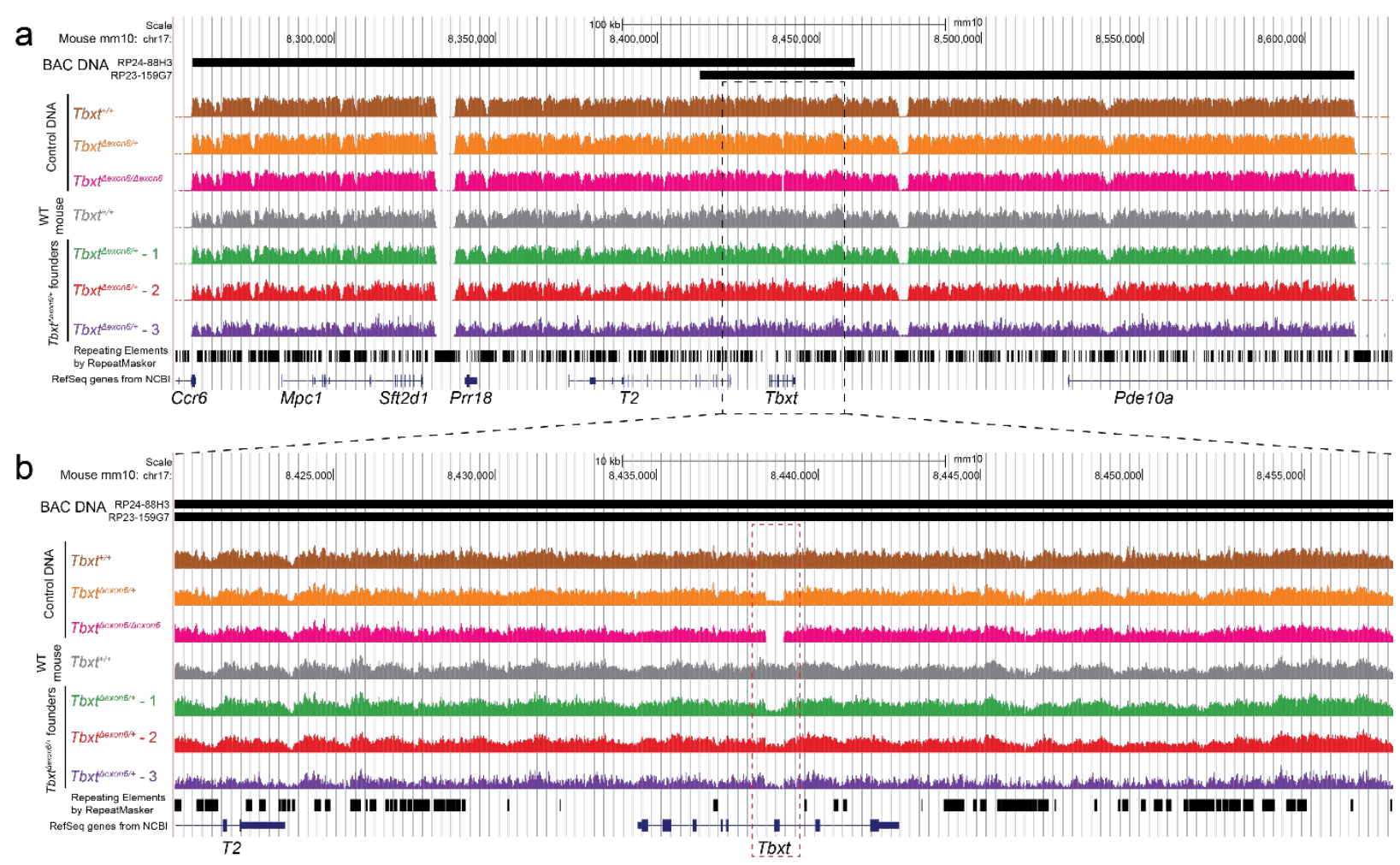

2 Fig. S6 | Capture-seq at the Tbxt locus of founder mice did not detect off target

mutations. a, Capture-seq of the founder mice using baits generated from bacterial artificial

chromosomes (RP24-88H3 and RP23-159G7). The shallow-covered regions are typically repeat sequences in the mouse genome and are consistent across samples. Control DNAs were obtained from wild-type or exon6-deleted mESCs through CRISPR targeting. b, A zoom-in view of the Capture-seq results at the Tbxt locus, highlighting the CRISPR-deleted exon 6 region. 


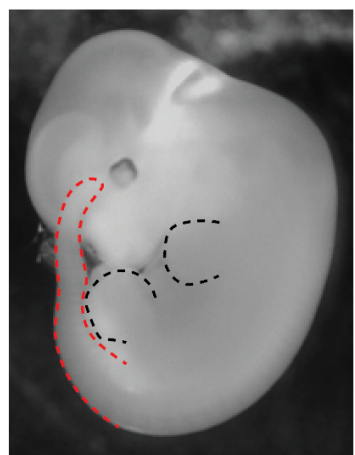

2

Tbxt $^{+/+}$

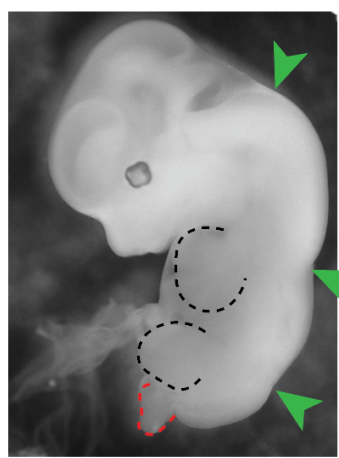

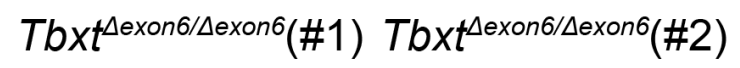

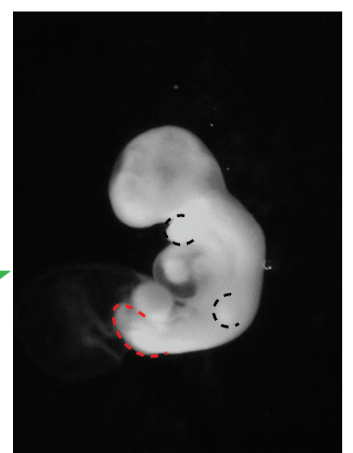

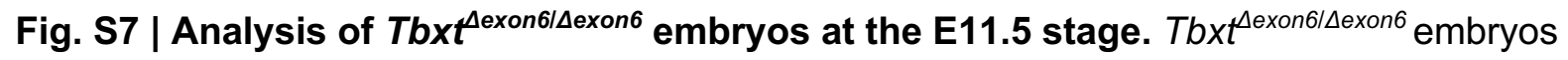
either develop spinal cord defects (middle) that die at birth or arrest at approximately stage E9 of development (right). Red and black dashed lines mark the embryonic tail regions and limb buds, respectively. Green arrowheads in the middle panel indicate malformed spinal cord regions. 


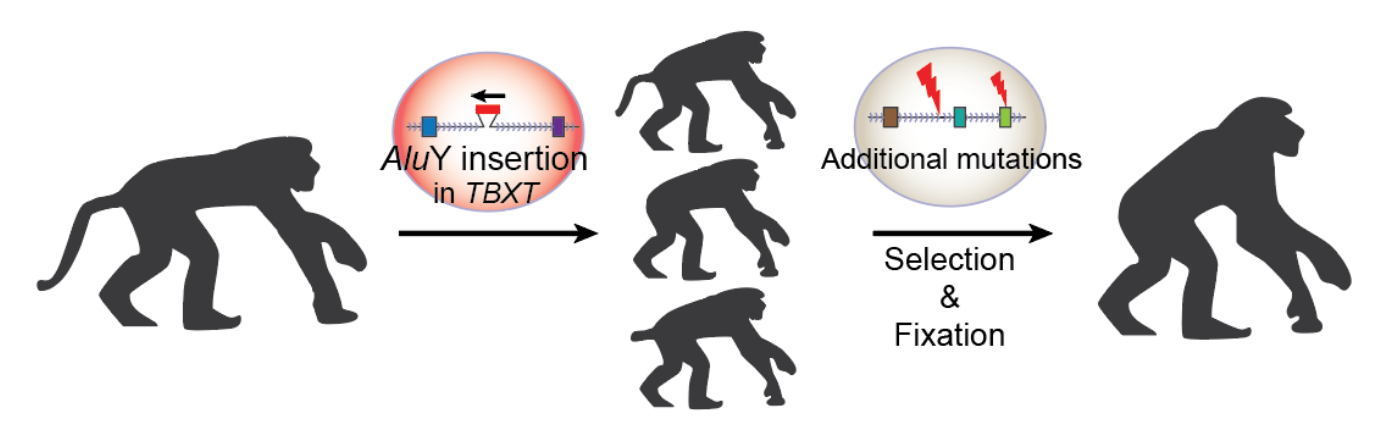

2 Fig. S8 | A model for tail-loss evolution in the early hominoids. The AluY insertion in TBXT marked an early genetic event that initiated tail-loss evolution in the hominoid common

4 ancestor. Additional genetic changes may have then acted to stabilize the no-tail phenotype in the ancient hominoids. 


\section{References}

2 1. Darwin, C. The descent of man, and selection in relation to sex. (degruyter.com, 2008).

2. Human evolution: an introduction to man's adaptations. (Routledge, 2017).

4 doi:10.4324/9780203789537

3. Hunt, K. D. The evolution of human bipedality: ecology and functional morphology. J. Hum. Evol. 26, 183-202 (1994).

4. Williams, S. A. \& Russo, G. A. Evolution of the hominoid vertebral column: The long and the short of it. Evol Anthropol 24, 15-32 (2015).

5. Hickman, G. C. The mammalian tail: a review of functions. Mamm. Rev. 9, 143-157 (1979).

6. Rogers, J. \& Gibbs, R. A. Comparative primate genomics: emerging patterns of genome content and dynamics. Nat. Rev. Genet. 15, 347-359 (2014).

7. Rhesus Macaque Genome Sequencing and Analysis Consortium et al. Evolutionary and biomedical insights from the rhesus macaque genome. Science 316, 222-234 (2007).

8. Chimpanzee Sequencing and Analysis Consortium. Initial sequence of the chimpanzee genome and comparison with the human genome. Nature 437, 69-87 (2005).

9. Kimelman, D. Tales of tails (and trunks): forming the posterior body in vertebrate embryos. Curr Top Dev Biol 116, 517-536 (2016).

10. Herrmann, B. G., Labeit, S., Poustka, A., King, T. R. \& Lehrach, H. Cloning of the T gene required in mesoderm formation in the mouse. Nature 343, 617-622 (1990).

11. Edwards, Y. H. et al. The human homolog T of the mouse T(Brachyury) gene; gene structure, cDNA sequence, and assignment to chromosome 6q27. Genome Res. 6, 226233 (1996).

12. Kispert, A., Koschorz, B. \& Herrmann, B. G. The T protein encoded by Brachyury is a tissue-specific transcription factor. EMBO J. 14, 4763-4772 (1995).

13. Wilde, J. J., Petersen, J. R. \& Niswander, L. Genetic, epigenetic, and environmental contributions to neural tube closure. Annu. Rev. Genet. 48, 583-611 (2014).

14. Sehner, S., Fichtel, C. \& Kappeler, P. M. Primate tails: Ancestral state reconstruction and determinants of interspecific variation in primate tail length. Am. J. Phys. Anthropol. 167, 750-759 (2018).

15. Russo, G. A. Postsacral vertebral morphology in relation to tail length among primates and other mammals. Anat Rec (Hoboken) 298, 354-375 (2015).

16. Lemelin, P. Comparative and functional myology of the prehensile tail in New World monkeys. J Morphol 224, 351-368 (1995).

17. Narita, Y. \& Kuratani, S. Evolution of the vertebral formulae in mammals: a perspective on developmental constraints. J Exp Zool B Mol Dev Evol 304, 91-106 (2005). 
18. Young, N. M., Wagner, G. P. \& Hallgrímsson, B. Development and the evolvability of human limbs. Proc. Natl. Acad. Sci. USA 107, 3400-3405 (2010).

19. Pontzer, H., Raichlen, D. A. \& Rodman, P. S. Bipedal and quadrupedal locomotion in chimpanzees. J. Hum. Evol. 66, 64-82 (2014).

20. Bauer, H. R. Chimpanzee bipedal locomotion in the Gombe National Park, East Africa. Primates (1977).

21. Mallo, M. The vertebrate tail: a gene playground for evolution. Cell Mol. Life Sci. 77, 10211030 (2020).

22. Smith, C. L. \& Eppig, J. T. The mammalian phenotype ontology: enabling robust annotation and comparative analysis. Wiley Interdiscip Rev Syst Biol Med 1, 390-399 (2009).

23. Wilkinson, D. G., Bhatt, S. \& Herrmann, B. G. Expression pattern of the mouse T gene and its role in mesoderm formation. Nature 343, 657-659 (1990).

24. Yamaguchi, T. P., Takada, S., Yoshikawa, Y., Wu, N. \& McMahon, A. P. T (Brachyury) is a direct target of Wnt3a during paraxial mesoderm specification. Genes Dev. 13, 3185-3190 (1999).

25. Tosic, J. et al. Eomes and Brachyury control pluripotency exit and germ-layer segregation by changing the chromatin state. Nat. Cell Biol. 21, 1518-1531 (2019).

26. Buckingham, K. J. et al. Multiple mutant T alleles cause haploinsufficiency of Brachyury and short tails in Manx cats. Mamm. Genome 24, 400-408 (2013).

27. Haworth, K. et al. Canine homolog of the T-box transcription factor T; failure of the protein to bind to its DNA target leads to a short-tail phenotype. Mamm. Genome 12, 212-218 (2001).

28. Schulte-Merker, S., van Eeden, F. J., Halpern, M. E., Kimmel, C. B. \& Nüsslein-Volhard, C. no tail (ntl) is the zebrafish homologue of the mouse T (Brachyury) gene. Development 120, 1009-1015 (1994).

29. Batzer, M. A. \& Deininger, P. L. Alu repeats and human genomic diversity. Nat. Rev. Genet. 3, 370-379 (2002).

30. Wallace, M. R. et al. A de novo Alu insertion results in neurofibromatosis type 1. Nature 353, 864-866 (1991).

31. Lev-Maor, G. et al. Intronic Alus influence alternative splicing. PLoS Genet. 4, e1000204 (2008).

32. Payer, L. M. et al. Alu insertion variants alter mRNA splicing. Nucleic Acids Res. 47, 421431 (2019).

33. Lorenz, R. et al. ViennaRNA Package 2.0. Algorithms Mol Biol 6, 26 (2011).

34. Xi, H. et al. In Vivo Human Somitogenesis Guides Somite Development from hPSCs. Cell Rep. 18, 1573-1585 (2017). 
35. Pour, M. et al. Emergence and patterning dynamics of mouse definitive endoderm. SSRN Journal (2021). doi:10.2139/ssrn.3848112

36. Kent, W. J. et al. The human genome browser at UCSC. Genome Res. 12, 996-1006 (2002).

37. Brosh, R. et al. A versatile platform for locus-scale genome rewriting and verification. Proc. Natl. Acad. Sci. USA 118, (2021).

38. International Human Genome Sequencing Consortium et al. Initial sequencing and analysis of the human genome. Nature 409, 860-921 (2001).

39. Jeck, W. R. et al. Circular RNAs are abundant, conserved, and associated with ALU repeats. RNA 19, 141-157 (2013).

40. Shaheen, R. et al. T (brachyury) is linked to a Mendelian form of neural tube defects in humans. Hum. Genet. 134, 1139-1141 (2015).

41. Shields, D. C. et al. Association between historically high frequencies of neural tube defects and the human T homologue of mouse T (Brachyury). Am. J. Med. Genet. 92, 206-211 (2000).

42. Morrison, K. et al. Genetic mapping of the human homologue ( $\mathrm{T}$ ) of mouse T(Brachyury) and a search for allele association between human T and spina bifida. Hum. Mol. Genet. 5, 669-674 (1996).

43. Postma, A. V. et al. Mutations in the T (brachyury) gene cause a novel syndrome consisting of sacral agenesis, abnormal ossification of the vertebral bodies and a persistent notochordal canal. J. Med. Genet. 51, 90-97 (2014).

44. Kumar, S., Stecher, G., Li, M., Knyaz, C. \& Tamura, K. MEGA X: Molecular evolutionary genetics analysis across computing platforms. Mol. Biol. Evol. 35, 1547-1549 (2018).

45. Herrero, J. et al. Ensembl comparative genomics resources. Database (Oxford) 2016, (2016).

46. Pour, M. et al. Emergence and patterning dynamics of mouse definitive endoderm. SSRN Journal (2021). doi:10.2139/ssrn.3848112

47. Concordet, J.-P. \& Haeussler, M. CRISPOR: intuitive guide selection for CRISPR/Cas9 genome editing experiments and screens. Nucleic Acids Res. 46, W242-W245 (2018).

48. Chen, F. et al. High-frequency genome editing using ssDNA oligonucleotides with zincfinger nucleases. Nat. Methods 8, 753-755 (2011). CRISPR/Cas-mediated genome engineering. Nat. Protoc. 9, 1956-1968 (2014). 